\title{
Synthesis of function generating spherical four bar mechanism for the six independent parameters
}

\author{
Rasim Alizade*, Erkin Gezgin \\ Mechanical Engineering Department, Izmir Institute of Technology, 35437 Izmir, Turkey
}

\section{A R T I C L E I N F O}

\section{Article history:}

Received 25 December 2008

Received in revised form 31 March 2011

Accepted 3 April 2011

Available online 13 May 2011

\section{Keywords:}

Spherical mechanisms

Synthesis

Design and precision points

Interpolation

Least squares

Chebyshev approximation

\begin{abstract}
A B S T R A C T
This study deals with the function generation synthesis of spherical four bar mechanism for six independent construction parameters $\varphi_{0}, \psi_{0}, \alpha_{1}, \alpha_{2}, \alpha_{3}$, and $\alpha_{4}$ by giving six or more design points with respect to the methods that are used in synthesis procedure. Quaternion algebra is used to derive the objective function of spherical four bar mechanism by following some rotational sequences. Three different methods as interpolation approximation, least squares approximation and Chebyshev approximation are used during synthesis procedure. During the consecutive trials in Chebyshev approximation, a new approach is taken to renew the design points $\varphi_{i}$ by plotting the graph of the objective functions derivative and taking the roots of it as new design points with two boundary points. Separate examples are given for each section and the results are tabulated. Discussions about the study and comparisons between the used methods are given at the end of the study.
\end{abstract}

(c) 2011 Elsevier Ltd. All rights reserved.

\section{Introduction}

In the design process of mechanisms, the importance of the kinematic synthesis problem cannot be neglected. After the selection of the mechanism that will be used for a specific task, its construction parameters must be designed with respect to the constraint conditions. Function generation is one of the synthesis kinds, where these conditions are related with some function between the input and output links. It is important that the whole calculation process can become complicated especially when the number of design parameters is increased. So that, different methods throughout the literature have been developed and tried in various mechanisms to overcome this difficulty.

As spherical mechanisms hold a transition position between the planar and spatial linkages, they attract many authors for synthesis purposes. Being the smallest member of close loop spherical mechanisms, function generation synthesis of four bar spherical linkage can be seen in many studies. Denavit and Hartenberg [1] presented the synthesis procedure for three precision points in the function generation of spherical four bar mechanism, where a logarithmic function is decided to be generated. Zimmerman [2] proposed an algorithm for the same mechanism for four precision points. Polynomial approximation is used for three, four and five precision points in the works of Alizade [3], Alizade and Kilit [4], Farhang et al. [5,6], Rao et al. [7], and Murray and McCarthy [8] for the spherical four bar mechanism. Also in the paper of Alizade and Kilit [4], effects of the locations of the precision points are considered and a graphical method in CAD environment is shown to verify the solutions of the construction parameters. Sancribrian et al. [9] proposed a synthesis method that uses a dimensional synthesis technique and local optimization, and Cervantes-Sanchez et al. [10] introduced a new approach for three and four precision points exact kinematic synthesis, where several examples for the spherical four bar mechanism are given. Also, Kazerounian and Solecki [11] and Gupta and Beloui [12] presented additional conditions as rotability, branch and circuit defect elimination etc. that can be controlled after the synthesis problem.

\footnotetext{
* Corresponding author. Tel.: +90232750 6782; fax: +902327506701.

E-mail addresses: alizada_rasim@hotmail.com (R. Alizade), erkingezgin@iyte.edu.tr (E. Gezgin).
} 


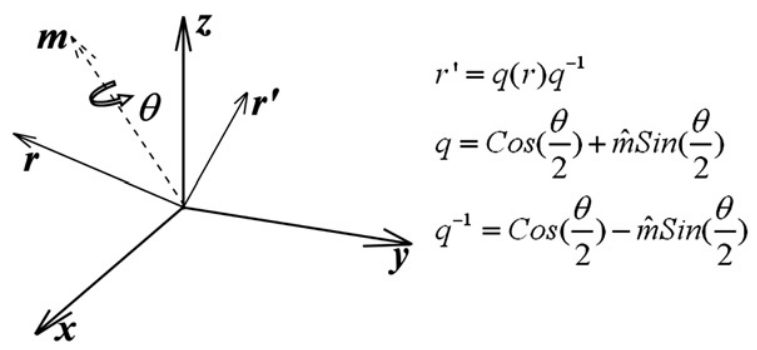

Fig. 1. Rotation of a vector by using quaternion operator.

The objective of current study is the function generation synthesis of spherical four bar mechanism for six independent construction parameters $\left(\varphi_{0}, \psi_{0}, \alpha_{1}, \alpha_{2}, \alpha_{3}, \alpha_{4}\right)$. Objective function of the mechanism is derived by using quaternion algebra. During synthesis, three different methods as interpolation approximation, least squares approximation and Chebyshev approximation are used and the differences between each other are compared.

\section{Objective function}

The main problem in the synthesis of any mechanism is the fact that, the objective function of the mechanism that will be synthesized should be found and simplified by using appropriate algebraic method. Due to common intersection point of all the joint axes in a spherical mechanism, quaternion algebra can be used as a great tool for this purpose. Note that, any quaternion operator $q() q^{-1}$ will rotate any vector around any axis by the desired amount of angle provided that they are passing from a common point (Fig. 1).

Consider that two unit vectors of the input and output joint axes in spherical four bar mechanism is selected as $\hat{\mathbf{r}}_{1}$ and $\hat{\mathbf{r}}_{\mathbf{4}}$, and the coordinate system is placed so that $\hat{\mathbf{r}}_{1}=\mathbf{i}$ (Fig. 2 ).

Starting from $\hat{\mathbf{r}}_{\mathbf{1}}, \hat{\mathbf{r}}_{\mathbf{4}}$ can be reached by using two distinct routes. The first one includes the construction parameters $\alpha_{1}, \alpha_{2}$ and $\alpha_{3}$ where $\hat{\mathbf{r}}_{\mathbf{1}}$ is rotated around the normal of $A O B$ plane $\mathbf{N}_{\mathbf{1}}$ by the angle $\alpha_{1}$ to reach $\hat{\mathbf{r}}_{\mathbf{2}}$, than $\hat{\mathbf{r}}_{\mathbf{2}}$ is rotated around the normal of $B O C$ plane $\mathbf{N}_{\mathbf{2}}$ by the angle $\alpha_{2}$ to reach $\hat{\mathbf{r}}_{\mathbf{3}}$, and finally $\hat{\mathbf{r}}_{\mathbf{3}}$ is rotated around the normal of COD plane $\mathbf{N}_{\mathbf{3}}$ by the angle $\alpha_{3}$ to reach $\hat{\mathbf{r}}_{\mathbf{4}}$ (Eq. (1)). The second one includes the construction parameter $\alpha_{4}$ where $\hat{\mathbf{r}}_{\mathbf{1}}$ is rotated around $z$ axis by the angle $\alpha_{4}$ to reach $\hat{\mathbf{r}}_{\mathbf{4}}$ (Eq. (2)).

$$
\begin{aligned}
\hat{\mathbf{r}}_{4} & =q_{3} q_{2} q_{1}\left(\hat{\mathbf{r}}_{1}\right) q_{1}^{-1} q_{2}^{-1} q_{3}^{-1} \\
q_{1} & =\operatorname{Cos}\left(\frac{\alpha_{1}}{2}\right)+\hat{\mathbf{n}}_{1} \operatorname{Sin}\left(\frac{\alpha_{1}}{2}\right), q_{2}=\operatorname{Cos}\left(\frac{\alpha_{2}}{2}\right)+\hat{\mathbf{n}}_{2} \operatorname{Sin}\left(\frac{\alpha_{2}}{2}\right), q_{3}=\operatorname{Cos}\left(\frac{\alpha_{3}}{2}\right)+\hat{\mathbf{n}}_{3} \operatorname{Sin}\left(\frac{\alpha_{3}}{2}\right) \\
\hat{\mathbf{r}}_{\mathbf{4}} & =q_{4}\left(\hat{\mathbf{r}}_{1}\right) q_{4}^{-1}, q_{4}=\operatorname{Cos}\left(\frac{\alpha_{4}}{2}\right)+\mathbf{k} \operatorname{Sin}\left(\frac{\alpha_{4}}{2}\right) .
\end{aligned}
$$

From this point, to make the calculations possible, $\hat{\mathbf{n}}_{\mathbf{1}}, \hat{\mathbf{n}}_{2}$ and $\hat{\mathbf{n}}_{3}$ should be defined clearly.

$\hat{\mathbf{n}}_{1}$ can be reached by rotating the unit vector of $z$ axis "k" around $x$ axis by the input angle $\varphi$ (Eq. (3)), $\hat{\mathbf{n}}_{2}$ can be reached by rotating $\hat{\mathbf{n}}_{1}$ around $\hat{\mathbf{r}}_{2}$ axis by the angle $\gamma$ (Eq. (4)), and $\hat{\mathbf{n}}_{3}$ can be reached by rotating the negative unit vector of $z$ axis " $-\mathbf{k}$ " around $\hat{\mathbf{r}}_{\mathbf{4}}$ axis by the output angle $\psi$ (Eq. (5)).

$$
\hat{\mathbf{n}}_{\mathbf{1}}=q_{5}(\mathbf{k}) q_{5}^{-1}, q_{5}=\operatorname{Cos}\left(\frac{\varphi}{2}\right)+\mathbf{i} \operatorname{Sin}\left(\frac{\varphi}{2}\right)
$$

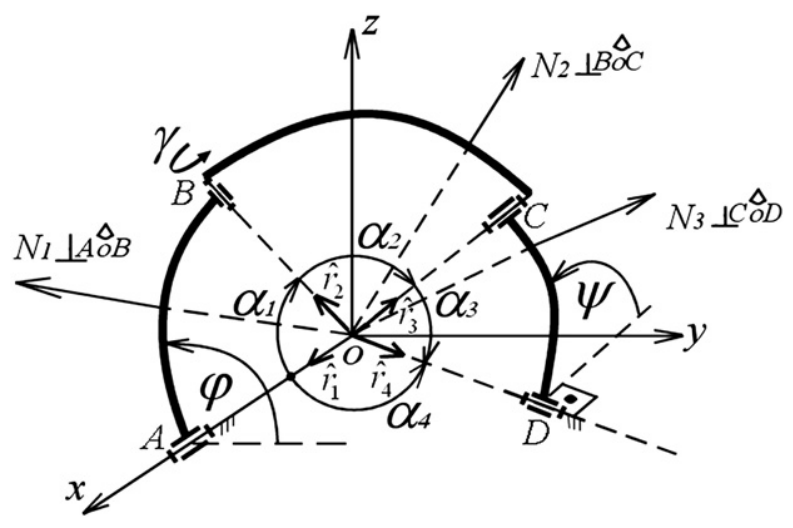

Fig. 2. Spherical four bar mechanism. 


$$
\begin{aligned}
& \hat{\mathbf{n}}_{\mathbf{2}}=q_{6}\left(\hat{\mathbf{n}}_{1}\right) q_{6}^{-1}, q_{6}=\operatorname{Cos}\left(\frac{\gamma}{2}\right)+\hat{\mathbf{r}}_{\mathbf{2}} \operatorname{Sin}\left(\frac{\gamma}{2}\right), \hat{\mathbf{r}}_{\mathbf{2}}=q_{1}\left(\hat{\mathbf{r}}_{\mathbf{1}}\right) q_{1}^{-1} \\
& \hat{\mathbf{n}}_{\mathbf{3}}=q_{7}(-\mathbf{k}) q_{7}^{-1}, q_{7}=\operatorname{Cos}\left(\frac{\psi}{2}\right)+\hat{\mathbf{r}}_{\mathbf{4}} \operatorname{Sin}\left(\frac{\psi}{2}\right), \hat{\mathbf{r}}_{\mathbf{4}}=q_{4}\left(\hat{\mathbf{r}}_{\mathbf{1}}\right) q_{4}^{-1}
\end{aligned}
$$

Defining $\hat{\mathbf{n}}_{\mathbf{1}}, \hat{\mathbf{n}}_{2}$ and $\hat{\mathbf{n}}_{3}$, Eqs. (1) and (2) can be recalled. Both of the equations result in a quaternion with null scalar part and three vector components $\mathbf{i}, \mathbf{j}$ and $\mathbf{k}$. If the vector components of two resulting quaternions are equalized, three equations, including construction parameters $\left(\alpha_{1}, \alpha_{2}, \alpha_{3}, \alpha_{4}\right)$, input parameter $(\varphi)$, and output parameter $(\psi)$, will be reached. However, these equations also include $\gamma$, in the form of $\operatorname{Cos}(\gamma)$ and $\operatorname{Sin}(\gamma)$ as variable parameters. Owing to the fact that $\gamma$ is neither construction nor input-output parameter, it is not needed during the function generation synthesis problem and it should be eliminated. In the light of this, using algebraic operations and manipulations, three equations are reduced into one equation that is free of $\gamma$. After the simplification of this equation, the objective function of spherical four bar mechanism is attained as,

$$
C \alpha_{2}-C \alpha_{1} C \alpha_{3} C \alpha_{4}+C \alpha_{1} C \psi S \alpha_{3} S \alpha_{4}-S \alpha_{1} C \alpha_{4} C \psi C \varphi S \alpha_{3}-S \alpha_{1} C \alpha_{3} C \varphi S \alpha_{4}-S \alpha_{1} S \alpha_{3} S \psi S \varphi=0
$$

where, $S$ and $C$ stand for sine and cosine of the angles respectively. On the other hand, when Eq. (6) is inspected, it can be seen that there exist only four design parameters $\left(\alpha_{1}, \alpha_{2}, \alpha_{3}, \alpha_{4}\right)$. Hence, the pole positions of the input link $\varphi_{0}$ and the output link $\psi_{0}$ is selected to fulfill the place of the remaining two parameters. When $\varphi$ and $\psi$ are replaced with $\left(\varphi_{0}+\varphi_{i}\right)$ and $\left(\psi_{0}+\psi_{i}\right)$ respectively in Eq. (6), the new objective function of the six independent parameters function generation synthesis of spherical four bar mechanism in open form will become,

$$
\begin{aligned}
C \alpha_{2} & -C \alpha_{1} C \alpha_{3} C \alpha_{4}+C \alpha_{1} S \alpha_{3} S \alpha_{4} C \psi_{0} C \psi_{i}-C \alpha_{1} S \alpha_{3} S \alpha_{4} S \psi_{0} S \psi_{i}-C \alpha_{3} S \alpha_{1} S \alpha_{4} C \varphi_{0} C \varphi_{i}+C \alpha_{3} S \alpha_{1} S \alpha_{4} S \varphi_{0} S \varphi_{i} \\
& -C \alpha_{4} S \alpha_{1} S \alpha_{3} C \psi_{0} C \varphi_{0} C \psi_{i} C \varphi_{i}+S \alpha_{1} S \alpha_{3} S \psi_{0} S \varphi_{0} C \psi_{i} C \varphi_{i}-S \alpha_{1} S \alpha_{3} C \psi_{0} C \varphi_{0} S \psi_{i} S \varphi_{i}+C \alpha_{4} S \alpha_{1} S \alpha_{3} S \psi_{0} S \varphi_{0} S \psi_{i} S \varphi_{i} \\
& +C \alpha_{4} S \alpha_{1} S \alpha_{3} C \varphi_{0} S \psi_{0} C \varphi_{i} S \psi_{i}-S \alpha_{1} S \alpha_{3} C \psi_{0} S \varphi_{0} C \varphi_{i} S \psi_{i}+C \alpha_{4} S \alpha_{1} S \alpha_{3} C \psi_{0} S \varphi_{0} C \psi_{i} S \varphi_{i}-S \alpha_{1} S \alpha_{3} C \varphi_{0} S \psi_{0} C \psi_{i} S \varphi_{i}=0
\end{aligned}
$$

Note that the new objective function (set of equationsi $=1,2, \ldots, 6)$ includes six independent parameters $\left(\varphi_{0}, \psi_{0}, \alpha_{1}, \alpha_{2}, \alpha_{3}, \alpha_{4}\right)$ to design.

\section{Interpolation approximation}

Before proceeding further, dividing both sides by $S \alpha_{1} S \alpha_{3} S \psi_{0} S \varphi_{0}$ Eq. (7) can be rewritten in a polynomial form as,

$$
\sum_{j=0}^{11} P_{j} f_{j}^{i}-F_{i}=0,(i=1,2, . ., 6)
$$

where, the constant parameters are,

$$
\begin{aligned}
& P_{0}=\left(C \alpha_{2}-C \alpha_{1} C \alpha_{3} C \alpha_{4}\right) /\left(S \alpha_{1} S \alpha_{3} S \psi_{0} S \varphi_{0}\right), P_{1}=-C \alpha_{4}, P_{2}=-\operatorname{Cot} \psi_{0}, P_{3}=-\operatorname{Cot} \alpha_{1} S \alpha_{4} / S \varphi_{0} \\
& P_{4}=\operatorname{Cot} \alpha_{3} S \alpha_{4} / S \psi_{0}, P_{5}=-\operatorname{Cot} \varphi_{0}, P_{6}=P_{2} P_{3}, P_{7}=P_{4} P_{5}, P_{8}=P_{1} P_{2} P_{5}, P_{9}=P_{1} P_{2}, P_{10}=-P_{2} P_{5} \\
& P_{11}=P_{1} P_{5}
\end{aligned}
$$

and the continuous independent functions are,

$$
\begin{aligned}
& f_{0}^{i}=1, f_{1}^{i}=S \psi_{i} S \varphi_{i}, f_{2}^{i}=S \psi_{i} C \varphi_{i}, f_{3}^{i}=S \psi_{i}, f_{4}^{i}=S \varphi_{i}, f_{5}^{i}=C \psi_{i} S \varphi_{i}, f_{6}^{i}=C \psi_{i}, f_{7}^{i}=C \varphi_{i} \\
& f_{8}^{i}=C \psi_{i} C \varphi_{i}, f_{9}^{i}=f_{5}^{i}, f_{10}^{i}=f_{1}^{i}, f_{11}^{i}=f_{2}^{i}, F_{i}=f_{8}^{i},(i=1,2, . ., 6) .
\end{aligned}
$$

It is apparent that, in Eq. (8), there exist six unknowns $\left\{P_{j}\right\}_{0}^{5}$, and six nonlinear terms $\left\{P_{j}\right\}_{6}^{11}$. If the nonlinear terms are rewritten in terms of nonlinear operators $\left\{\lambda_{k}\right\}_{1}^{6}$, a new set of equations will be reached as,

$$
\begin{array}{r}
P_{2} P_{3}-\lambda_{1}=0, P_{4} P_{5}-\lambda_{2}=0 \\
P_{1} P_{2} P_{5}-\lambda_{3}=0, P_{1} P_{2}-\lambda_{4}=0 \\
-P_{2} P_{5}-\lambda_{5}=0, P_{1} P_{5}-\lambda_{6}=0
\end{array}
$$

where, $P_{6}=\lambda_{1}, P_{7}=\lambda_{2}, P_{8}=\lambda_{3}, P_{9}=\lambda_{4}, P_{10}=\lambda_{5}, P_{11}=\lambda_{6}$. If the terms with nonlinear operators are gathered to the same side of the equation, Eq. (8) becomes,

$$
\sum_{j=0}^{5} P_{j} f_{j}^{i}=f_{8}^{i}-\lambda_{1} f_{6}^{i}-\lambda_{2} f_{7}^{i}-\lambda_{3} f_{8}^{i}-\lambda_{4} f_{5}^{i}-\lambda_{5} f_{1}^{i}-\lambda_{6} f_{2}^{i},(i=1,2, . ., 6) .
$$


Now, all the terms on the left side of Eq. (10) is linear, so that the unknown parameters $\left\{P_{j}\right\}_{0}^{5}$ can be assumed as linearly proportional with the nonlinear operators as,

$$
P_{j}=l_{j}+m_{j} \lambda_{1}+n_{j} \lambda_{2}+p_{j} \lambda_{3}+q_{j} \lambda_{4}+r_{j} \lambda_{5}+s_{j} \lambda_{6},(j=0,1, . ., 5)
$$

If Eq. (11) is inserted into Eq. (10), and the parameters with the same nonlinear operators are equalized, 42 equations with 42 unknowns $\left(l_{j}, m_{j}, n_{j}, p_{j}, q_{j}, r_{j}, s_{j} j=0,1, . .5\right)$ will be reached. These equations can be written in matrix form as below,

$$
\left[\begin{array}{ccccccc}
{[A]_{6 \times 6}} & \cdot & \cdot & \cdot & \cdot & \cdot & \cdot \\
\cdot & {[A]_{6 \times 6}} & \cdot & \cdot & \cdot & \cdot & \cdot \\
\cdot & \cdot & {[A]_{6 \times 6}} & \cdot & \cdot & \cdot & \cdot \\
\cdot & \cdot & \cdot & {[A]_{6 \times 6}} & \cdot & \cdot & \cdot \\
\cdot & \cdot & \cdot & \cdot & {[A]_{6 \times 6}} & \cdot & \cdot \\
\cdot & \cdot & \cdot & \cdot & \cdot & {[A]_{6 \times 6}} & \cdot \\
\cdot & \cdot & \cdot & \cdot & \cdot & \cdot & {[A]_{6 \times 6}}
\end{array}\right]\left[\begin{array}{c}
L \\
M \\
N \\
P \\
Q \\
R \\
S
\end{array}\right]=\left[\begin{array}{c}
T \\
U \\
V \\
W \\
Y \\
Z \\
G
\end{array}\right]
$$

where,

$$
\begin{aligned}
& T=\left[\left\{f_{8}^{i}\right\}_{1}^{6}\right]^{T}
\end{aligned}
$$

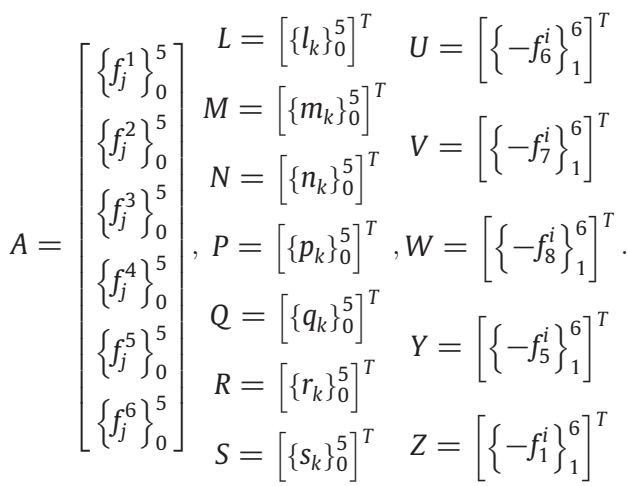

$$
\begin{aligned}
& G=\left[\left\{-f_{2}^{i}\right\}_{1}^{6}\right]^{T}
\end{aligned}
$$

Solution of Eq. (12) will give the unknowns $\left(l_{j}, m_{j}, n_{j}, p_{j}, q_{j}, r_{j}, s_{j} j=0,1, \ldots, 5\right)$. After substituting them into Eqs. (9) and (11), six nonlinear equations with six unknown nonlinear operators $\left\{\lambda_{k}\right\}_{1}^{6}$ are attained. Solving these equations numerically for nonlinear operators and inserting one of the real solutions into the Eq. (11), $\left\{P_{j}\right\}_{0}^{5}$ can be calculated and the six construction parameters will become,

$$
\begin{aligned}
& \psi_{0}=\operatorname{Cot}^{-1}\left(-P_{2}\right), \varphi_{0}=\operatorname{Cot}^{-1}\left(-P_{5}\right), \alpha_{4}=\operatorname{Cos}^{-1}\left(-P_{1}\right), \alpha_{1}=\operatorname{Cot}^{-1}\left(-P_{3} S \varphi_{0} / S \alpha_{4}\right) \\
& \alpha_{3}=\operatorname{Cot}^{-1}\left(P_{4} S \psi_{0} / S \alpha_{4}\right), \alpha_{2}=\operatorname{Cos}^{-1}\left(S \alpha_{1} S \alpha_{3} S \psi_{0} S \varphi_{0}+C \alpha_{1} C \alpha_{3} C \alpha_{4}\right) .
\end{aligned}
$$

\subsection{Numerical example}

In the case of numerical example, the input of the mechanism is decided to be selected from an interval of $2 \pi / 3<\varphi<4 \pi / 3$, the output function is selected as $\psi=\varphi^{0.8}$, and the six design points in prescribed interval are given with respect to the Chebyshev

\begin{tabular}{|c|c|c|c|c|c|c|}
\hline$i$ & 1 & 2 & 3 & 4 & 5 & 6 \\
\hline$\varphi_{i}(\mathrm{rad})$ & 2.13008 & 2.40111 & 2.87056 & 3.41263 & 3.88207 & 4.15311 \\
\hline \multirow[t]{2}{*}{$\psi_{i}(\mathrm{rad})$} & 1.83112 & 2.01525 & 2.32473 & 2.66975 & 2.95972 & 3.12391 \\
\hline & $\varphi_{0}(\mathrm{rad})$ & $\psi_{0}(\mathrm{rad})$ & $\alpha_{1}(\mathrm{rad})$ & $\alpha_{2}(\mathrm{rad})$ & $\alpha_{3}(\mathrm{rad})$ & $\alpha_{4}(\mathrm{rad})$ \\
\hline Construction parameters & 1.23867 & $-0.52161(5.76158)$ & 0.38103 & 1.32361 & $-1.49412(4.78907)$ & 0.17546 \\
\hline
\end{tabular}
spacing. The given design points and the calculated construction parameters are tabulated in Table 1. Also the designed spherical four bar mechanism and the plot of its objective function in the design interval can be seen in Fig. 3.

Table 1

Given precision points and calculated construction parameters for NE 3.1. 

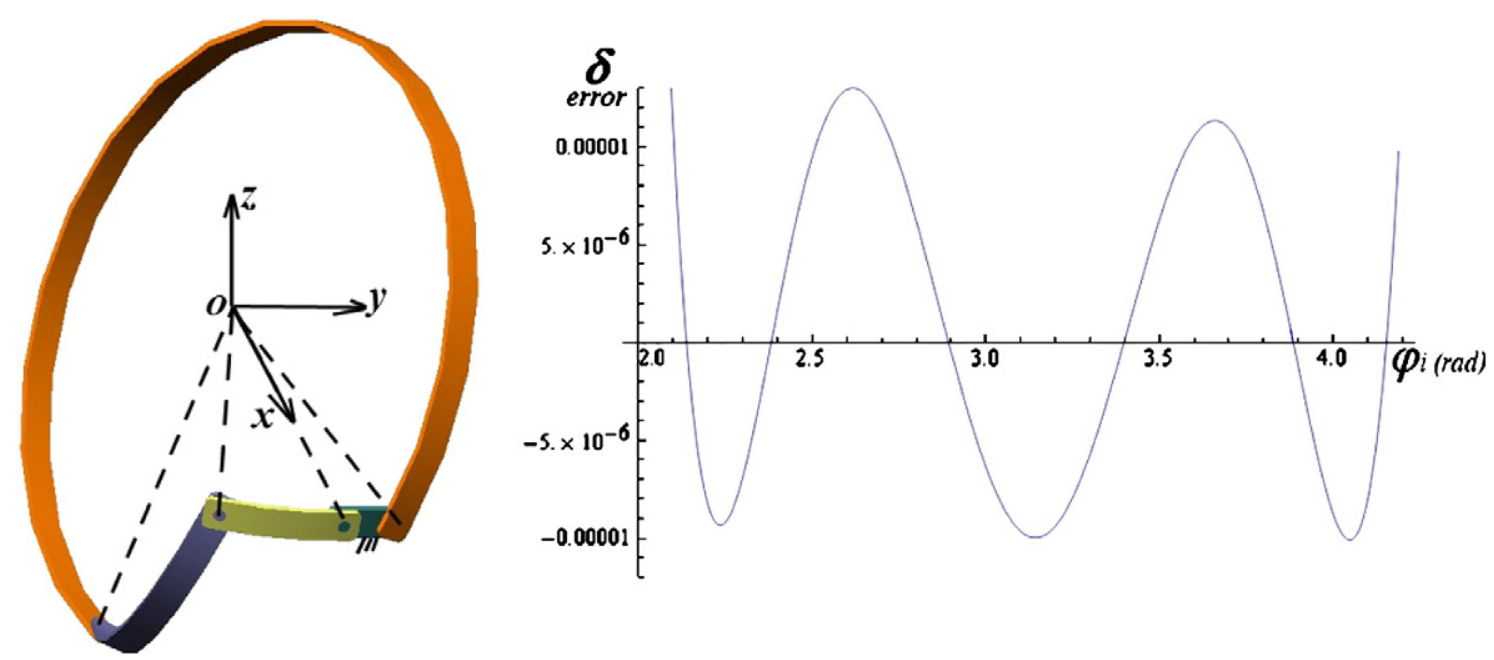

Fig. 3. Designed spherical four bar mechanism and its objective function.

Note that the objective function takes its zero values only at the six precision points thats are equal to the six design points as expected.Although the errors in intervals between the precision points seem to be inform, there are small deviations on the extremum vslues.

\section{Least square approximation}

In the previous section, the fitting error was $\delta=F\left(\varphi_{i}, \bar{c}\right)-F\left(\varphi_{i}\right)$, where $\bar{c}$ stands for the construction parameters, $F\left(\varphi_{i}\right)$ is the actual function, and $F\left(\varphi_{i}, \bar{c}\right)$ is the predicted function from the given design points. As a result, the fitting error vanishes at the six design points. However, in that specific example, number of design points that are given just equal to the number of unknown parameters. Now consider the case when the design points are over determined; that is, number of design points $n$ are greater than the number of construction parameters $m$.

The problem can be solved by using least square approximation method to find the best fitting function with respect to the given design point set. The least square approximation method suggests that, the best fitting function is reached when the sum of squared fitting errors $(\eta)$ is a minimum.

$$
\eta=\sum_{i=1}^{n} \delta^{2}=\sum_{i=1}^{n}\left(F\left(\varphi_{i}, \bar{c}\right)-F\left(\varphi_{i}\right)\right)^{2}
$$

The minimum $\eta$ is reached if and only if when the partial derivations of Eq. (14) with respect to the construction parameters are zero.

$$
\frac{\partial \eta}{\partial P_{j}}=0,(j=0,1, \ldots, l) .
$$

Using Eq. (15), $l+1$ equations $(l=m-1)$ are generated for the same amount of construction parameters and should be solved with respect to the given design conditions.

For the synthesis problem of this study, Eq. (14) can be rewritten as

$$
\eta=\sum_{i=1}^{n}\left(\sum_{j=0}^{11}\left(P_{j} f_{j}^{i}\right)-F_{i}\right)^{2}
$$

If Eq. (11) is inserted into Eqs. ((15), (16)), and the parameters with the same nonlinear operators are equalized, 42 equations with 42 unknowns can be written in matrix form,

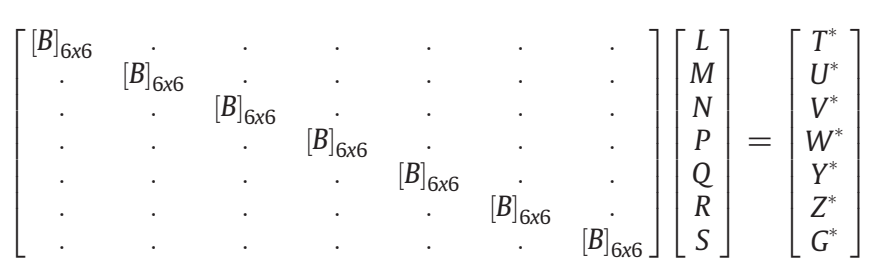


Table 2

Given precision points and calculated construction parameters for NE 4.1.

\begin{tabular}{|c|c|c|c|c|c|c|c|}
\hline$i$ & 1 & 2 & 3 & 4 & 5 & 6 & 7 \\
\hline$\varphi_{i}(\mathrm{rad})$ & 2.10098 & 2.15316 & 2.25491 & 2.40111 & 2.58445 & 2.79573 & 3.02434 \\
\hline$\psi_{i}(\mathrm{rad})$ & 1.81108 & 1.84698 & 1.91647 & 2.01525 & 2.13744 & 2.27612 & 2.42385 \\
\hline$i$ & 8 & 9 & 10 & 11 & 12 & 13 & 14 \\
\hline$\varphi_{i}(\mathrm{rad})$ & 3.25884 & 3.48746 & 3.69874 & 3.88207 & 4.02828 & 4.13002 & 4.18221 \\
\hline \multirow[t]{2}{*}{$\psi_{i}(\mathrm{rad})$} & 2.57306 & 2.71649 & 2.84736 & 2.95972 & 3.04857 & 3.11001 & 3.14141 \\
\hline & $\varphi_{0}(\mathrm{rad})$ & $\psi_{0}(\mathrm{rad})$ & $\alpha_{1}(\mathrm{rad})$ & $\alpha_{2}(\mathrm{rad})$ & $\alpha_{3}(\mathrm{rad})$ & $\alpha_{4}(\mathrm{rad})$ & \\
\hline Construction parameters & 1.24252 & $-0.51943(5.76376)$ & 0.38098 & 1.32458 & $-1.49467(4.78852)$ & 0.17518 & \\
\hline
\end{tabular}

where,

$$
B=\left[\begin{array}{ll}
T^{*}=\left[\left[f_{8}^{i} f_{0}^{i}\right]\left[f_{8}^{i} f_{1}^{i}\right]\left[f_{8}^{i} f_{2}^{i}\right]\left[f_{8}^{i} f_{3}^{i}\right]\left[f_{8}^{i} f_{4}^{i}\right]\left[f_{1}^{i}\right]\left[f_{8}^{i} f_{5}^{i} f_{2}^{i}\right]\left[f_{0}^{i} f_{3}^{i}\right]\left[f_{0}^{i} f_{4}^{i}\right]\left[f_{0}^{i} f_{5}^{i}\right]\right. \\
{\left[f_{1}^{i} f_{0}^{i}\right]\left[f_{1}^{i} f_{1}^{i}\right]\left[f_{1}^{i} f_{2}^{i}\right]\left[f_{1}^{i} f_{3}^{i}\right]\left[f_{1}^{i} f_{4}^{i}\right]\left[f_{1}^{i} f_{5}^{i}\right]} & \left.U^{*}=\left[f_{6}^{i} f_{0}^{i}\right]-\left[f_{6}^{i} f_{1}^{i}\right]-\left[f_{6}^{i} f_{2}^{i}\right]-\left[f_{6}^{i} f_{3}^{i}\right]-\left[f_{6}^{i} f_{4}^{i}\right]-\left[f_{6}^{i} f_{5}^{i}\right]\right]^{T} \\
{\left[f_{2}^{i} f_{0}^{i}\right]\left[f_{2}^{i} f_{1}^{i}\right]\left[f_{2}^{i} f_{2}^{i}\right]\left[f_{2}^{i} f_{3}^{i}\right]\left[f_{2}^{i} f_{4}^{i}\right]\left[f_{2}^{i} f_{5}^{i}\right]} & \left.V^{*}=\left[f_{7}^{i} f_{1}^{i}\right]-\left[f_{7}^{i} f_{2}^{i}\right]-\left[f_{7}^{i} f_{3}^{i}\right]-\left[f_{7}^{i} f_{4}^{i}\right]-\left[f_{7}^{i} f_{5}^{i}\right]\right]^{T} \\
{\left[f_{3}^{i} f_{0}^{i}\right]\left[f_{3}^{i} f_{1}^{i}\right]\left[f_{3}^{i} f_{2}^{i}\right]\left[f_{3}^{i} f_{3}^{i}\right]\left[f_{3}^{i} f_{4}^{i}\right]\left[f_{3}^{i} f_{5}^{i}\right]} & W^{*}=\left[-\left[f_{8}^{i} f_{0}^{i}\right]-\left[f_{8}^{i} f_{1}^{i}\right]-\left[f_{8}^{i} f_{2}^{i}\right]-\left[f_{8}^{i} f_{3}^{i}\right]-\left[f_{8}^{i} f_{4}^{i}\right]-\left[f_{8}^{i} f_{5}^{i}\right]\right]^{T} \text { and, }\left[f_{j}^{i} f_{k}^{i}\right]=\sum_{i=1}^{n} f_{j}^{i} f_{k}^{i} \\
{\left[f_{4}^{i} f_{0}^{i}\right]\left[f_{4}^{i} f_{1}^{i}\right]\left[f_{4}^{i} f_{2}^{i}\right]\left[f_{4}^{i} f_{3}^{i}\right]\left[f_{4}^{i} f_{4}^{i}\right]\left[f_{4}^{i} f_{5}^{i}\right]} & Y^{*}=\left[-\left[f_{5}^{i} f_{0}^{i}\right]-\left[f_{5}^{i} f_{1}^{i}\right]-\left[f_{5}^{i} f_{2}^{i}\right]-\left[f_{5}^{i} f_{3}^{i}\right]-\left[f_{5}^{i} f_{4}^{i}\right]-\left[f_{5}^{i} f_{5}^{i}\right]\right]^{T} \\
{\left[f_{5}^{i} f_{0}^{i}\right]\left[f_{5}^{i} f_{1}^{i}\right]\left[f_{5}^{i} f_{2}^{i}\right]\left[f_{5}^{i} f_{3}^{i}\right]\left[f_{5}^{i} f_{4}^{i}\right]\left[f_{5}^{i} f_{5}^{i}\right]} & Z^{*}=\left[-\left[f_{1}^{i} f_{0}^{i}\right]-\left[f_{1}^{i} f_{1}^{i}\right]-\left[f_{1}^{i} f_{2}^{i}\right]-\left[f_{1}^{i} f_{3}^{i}\right]-\left[f_{1}^{i} f_{4}^{i}\right]-\left[f_{1}^{i} f_{5}^{i}\right]\right]^{T} \\
G^{*}=\left[-\left[f_{2}^{i} f_{0}^{i}\right]-\left[f_{2}^{i} f_{1}^{i}\right]-\left[f_{2}^{i} f_{2}^{i}\right]-\left[f_{2}^{i} f_{3}^{i}\right]-\left[f_{2}^{i} f_{4}^{i}\right]-\left[f_{2}^{i} f_{5}^{i}\right]\right]^{T}
\end{array}\right.
$$

From this point, following the same analogy, the solution of Eq. (17) will give the unknowns $\left(l_{j}, m_{j}, n_{j}, p_{j}, q_{j}, r_{j}, s_{j} j=0,1, . ., 5\right)$. Inserting these parameters into Eqs. ((9) and (11)), and solving the equations numerically for nonlinear operators $\left.\left\{\lambda_{k}\right\}\right\}_{1}^{6}$, Eqs. (11) and (13) can be used to find construction parameters $\left(\varphi_{0}, \psi_{0}, \alpha_{1}, \alpha_{2}, \alpha_{3}, \alpha_{4}\right)$.

\subsection{Numerical example}

Using the same interval $(2 \pi / 3<\varphi<4 \pi / 3)$, and the output function $\left(\psi=\varphi^{0.8}\right)$, fourteen design points in prescribed interval is given again with respect to the Chebyshev spacing. The given design points and the calculated construction parameters are tabulated in Table 2. Also the plot of the designed mechanisms objective function and the sum of squared fitting errors $\eta$ with the mean of $\eta$ in the design interval can be seen in Fig. 4.

It is important to note that, although 14 design points are given, the fitting error vanishes only at six precision points. Also, when compared with the previous method, the values of the construction parameters $\left(\varphi_{0}, \psi_{0}, \alpha_{1}, \alpha_{2}, \alpha_{3}, \alpha_{4}\right)$ are close but different. This small difference on the other hand affects the extremum values of the fitting errors; that is, the max or min errors in intervals between the precision points are more uniform than the previous results.

\section{Chebyshev approximation}

Although the square approximation gives the reasonable results for the fitting error extremums in the design interval, the errors are not equal. Knowing that the best fitting error function, in this study the objective function, will be reached when it oscillates between an error bound of $\pm \Gamma$ with maximum absolute error $\Gamma$ in each sections in the design interval (Eq. (18)), Chebyshev approximation method is decided to be used to in the synthesis problem.

$$
\left|F\left(\varphi_{i}, \bar{c}\right)-F\left(\varphi_{i}\right)\right|=\Gamma,(i=1,2, \ldots, n) .
$$

Consider that the objective function is in the form of

$$
\sum_{j=0}^{l} P_{j} f_{j}+\sum_{j=l+1}^{l+k} P_{j} f_{j}-F=\delta
$$

where, $\mathrm{k}$ is the number of nonlinear parameters. The function in Eq. (19) consecutively changes sign $(l+2)$ times, so that it has $(l+2)$ extremums in the design interval, where two of them are the boundary precision points. Due to the fact that $\mathrm{max} / \mathrm{min}$ error value $\pm \Gamma$ is 
also being searched to bind the objective function at the given design points in the design interval, $\Gamma$ should be defined as a design parameter in synthesis equation that is again assumed to be linearly proportional with the nonlinear operators as,

$$
\Gamma=l_{6}+m_{6} \lambda_{1}+n_{6} \lambda_{2}+p_{6} \lambda_{3}+q_{6} \lambda_{4}+r_{6} \lambda_{5}+s_{6} \lambda_{6} .
$$

As a result, total number of parameters to be calculated with needed design points will increase by one, and Eq. (18) can be rewritten to form $n=l+2$ equations.

$$
\sum_{j=0}^{11} P_{j} f_{j}^{i}-F_{i}=(-1)^{i+1} \Gamma,(i=1,2, \ldots, 7) .
$$

If Eq. (11) is inserted into Eq. (21), and the parameters with the same nonlinear operators are equalized, 49 equations with 49 unknowns can be written in matrix form as

$$
\left[\begin{array}{ccccccc}
{[C]_{7 \times 7}} & \cdot & \cdot & \cdot & \cdot & \cdot & \cdot \\
\cdot & {[C]_{7 \times 7}} & \cdot & \cdot & \cdot & \cdot & \cdot \\
\cdot & \cdot & {[C]_{7 \times 7}} & \cdot & \cdot & \cdot & \cdot \\
\cdot & \cdot & \cdot & {[C]_{7 \times 7}} & \cdot & \cdot & \cdot \\
\cdot & \cdot & \cdot & \cdot & {[C]_{7 \times 7}} & \cdot & \cdot \\
\cdot & \cdot & \cdot & \cdot & \cdot & {[C]_{7 \times 7}} & \cdot \\
\cdot & \cdot & \cdot & \cdot & \cdot & \cdot & {[C]_{7 \times 7}}
\end{array}\right]\left[\begin{array}{c}
L^{*} \\
M^{*} \\
N^{*} \\
P^{*} \\
Q^{*} \\
R^{*} \\
S^{*}
\end{array}\right]=\left[\begin{array}{c}
T^{* *} \\
U^{* *} \\
V^{* *} \\
W^{* *} \\
Y^{* *} \\
Z^{* *} \\
G^{* *}
\end{array}\right]
$$

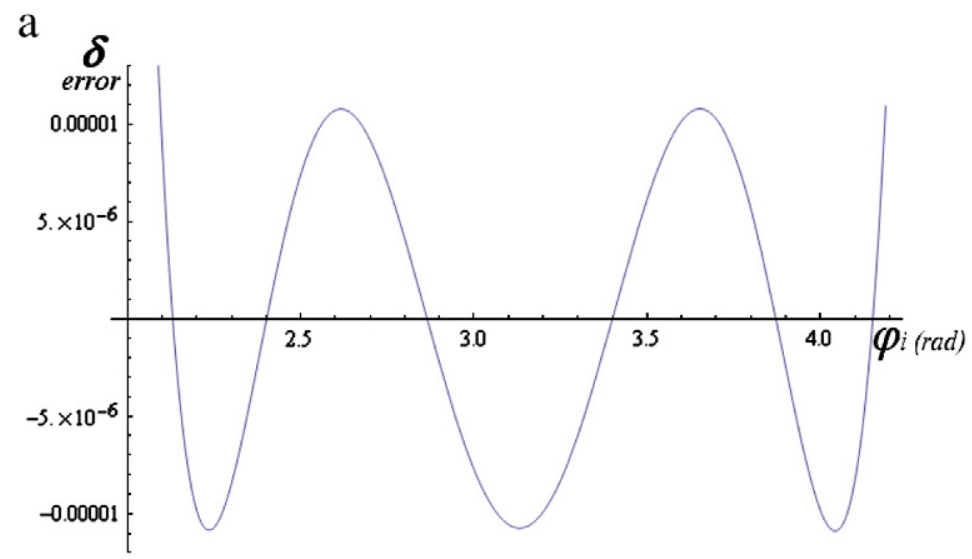

\section{$\mathrm{b}$}

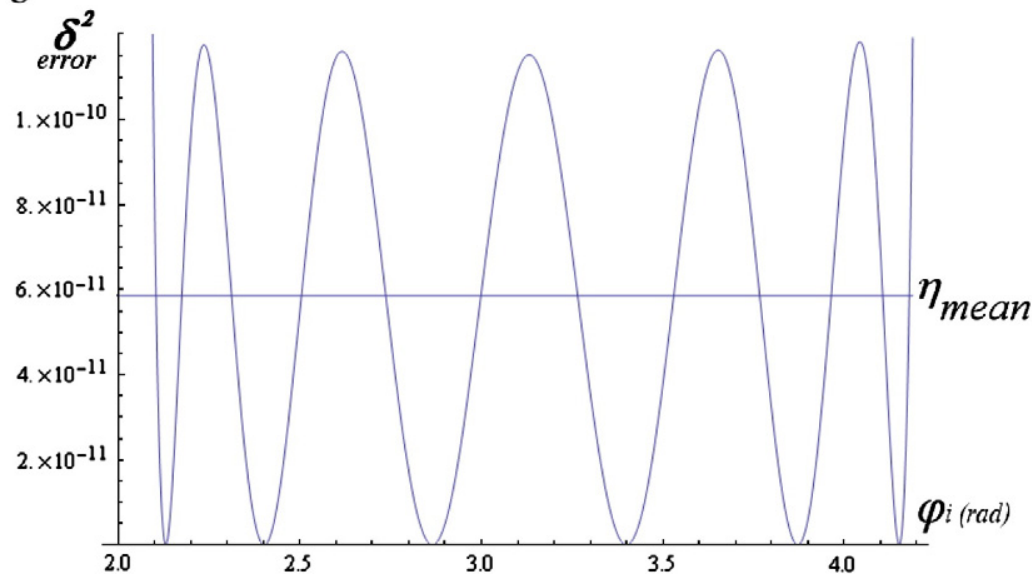

Fig. 4. a) Objective function of the designed spherical four bar mechanism and b) sum of squared fitting errors $\eta$ with $\eta_{\text {mean }}$. 
where,

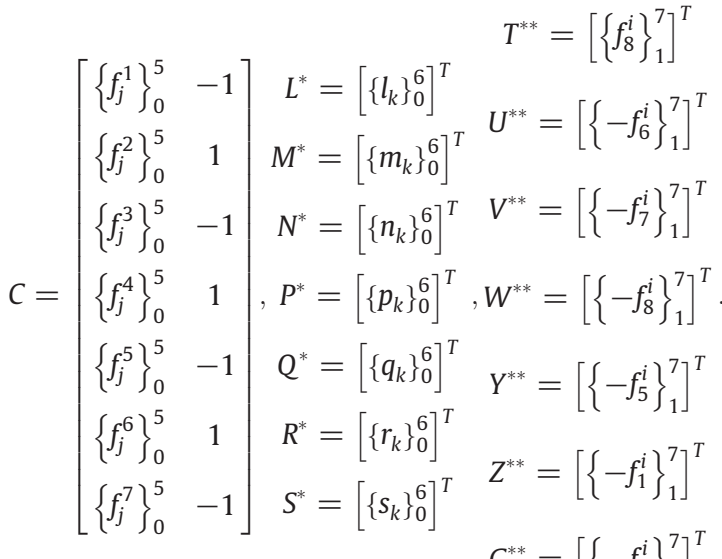

$$
\begin{aligned}
& G^{* *}=\left[\left\{-f_{2}^{i}\right\}_{1}^{7}\right]^{T}
\end{aligned}
$$

Using again the same analogy, the solution of Eq. (22) will give the unknowns $\left(l_{j}, m_{j}, n_{j}, p_{j}, q_{j}, r_{j}, s_{j} j=0,1, \ldots, 6\right)$. Inserting the unknowns into Eq. (9), and solving numerically for nonlinear operators $\left\{\lambda_{k}\right\}_{1}^{6}$, Eqs. ((11), (13), and 20) can be used to find construction parameters $\left(\varphi_{0}, \psi_{0}, \alpha_{1}, \alpha_{2}, \alpha_{3}, \alpha_{4}\right)$ and fitting error limit $\Gamma$. After the parameters are calculated, the derivative of Eq. (21) with respect to $\varphi_{i}$ is taken and its function is drawn in the design interval to find $(n-2)$ roots of the derivative equation. As the roots are the extremums of the generated objective function with the synthesized parameters, they will become new $\varphi_{i}$ values with the previous boundary precision points. Newer construction parameters and fitting error limit $\Gamma$ will be recalculated by using Eqs. ((9), (11), (13), (20), and (22)) in this order. This consecutive process should be continued until Eq. (18) is satisfied.

Table 3

Given precision points and calculated construction parameters for NE 5.1.

\begin{tabular}{|c|c|c|c|c|c|c|c|}
\hline Trial 1 & 1 & 2 & 3 & 4 & 5 & 6 & 7 \\
\hline$\varphi_{i}(\mathrm{rad})$ & 2.12065 & 2.32286 & 2.68723 & 3.14159 & 3.59595 & 3.96032 & 4.16253 \\
\hline \multirow[t]{2}{*}{$\psi_{i}(\mathrm{rad})$} & 1.82463 & 1.96254 & 2.20518 & 2.49873 & 2.78389 & 3.00735 & 3.12958 \\
\hline & $\varphi_{0}(\mathrm{rad})$ & $\psi_{0}(\mathrm{rad})$ & $\alpha_{1}(\mathrm{rad})$ & $\alpha_{2}(\mathrm{rad})$ & $\alpha_{3}(\mathrm{rad})$ & $\alpha_{4}(\mathrm{rad})$ & $\Gamma$ \\
\hline Construction parameters & 1.24133 & $\begin{array}{r}-0.52034 \\
(5.76285)\end{array}$ & 0.37989 & 1.32471 & $\begin{array}{l}-1.49455 \\
(4.78864)\end{array}$ & 0.17462 & $\begin{array}{l}8.48283 \\
10^{-6}\end{array}$ \\
\hline Trial 2 & 1 & 2 & 3 & 4 & 5 & 6 & 7 \\
\hline$\varphi_{i}(\mathrm{rad})$ & 2.12065 & 2.25868 & 2.63882 & 3.13495 & 3.63403 & 4.02038 & 4.16253 \\
\hline \multirow[t]{2}{*}{$\psi_{i}(\mathrm{rad})$} & 1.82463 & 1.91904 & 2.17335 & 2.49451 & 2.80745 & 3.04379 & 3.12958 \\
\hline & $\varphi_{0}(\mathrm{rad})$ & $\psi_{0}(\mathrm{rad})$ & $\alpha_{1}(\mathrm{rad})$ & $\alpha_{2}(\mathrm{rad})$ & $\alpha_{3}(\mathrm{rad})$ & $\alpha_{4}(\mathrm{rad})$ & $\Gamma$ \\
\hline Construction parameters & 1.24225 & $\begin{array}{r}-0.51978 \\
(5.76341)\end{array}$ & 0.38008 & 1.32487 & $\begin{array}{r}-1.49468 \\
(4.78851)\end{array}$ & 0.17467 & $\begin{array}{l}9.26939 \\
10^{-6}\end{array}$ \\
\hline Trial 3 & 1 & 2 & 3 & 4 & 5 & 6 & 7 \\
\hline$\varphi_{i}(\mathrm{rad})$ & 2.12065 & 2.25770 & 2.62865 & 3.13255 & 3.64097 & 4.02100 & 4.16253 \\
\hline \multirow[t]{2}{*}{$\psi_{i}(\mathrm{rad})$} & 1.82463 & 1.91838 & 2.16664 & 2.49298 & 2.81174 & 3.04416 & 3.12958 \\
\hline & $\varphi_{0}(\mathrm{rad})$ & $\psi_{0}(\mathrm{rad})$ & $\alpha_{1}(\mathrm{rad})$ & $\alpha_{2}(\mathrm{rad})$ & $\alpha_{3}(\mathrm{rad})$ & $\alpha_{4}(\mathrm{rad})$ & $\Gamma$ \\
\hline Construction parameters & 1.24227 & $\begin{array}{r}-0.51977 \\
(5.76342)\end{array}$ & 0.38008 & 1.32488 & $\begin{array}{r}-1.49468 \\
(4.78851)\end{array}$ & 0.17467 & $\begin{array}{l}9.27502 \\
10^{-6}\end{array}$ \\
\hline Trial 4 & 1 & 2 & 3 & 4 & 5 & 6 & 7 \\
\hline \multirow{3}{*}{$\begin{array}{l}\varphi_{i}(\mathrm{rad}) \\
\psi_{i}(\mathrm{rad})\end{array}$} & 2.12065 & 2.25775 & 2.62866 & 3.13247 & 3.64093 & 4.02096 & 4.16253 \\
\hline & 1.82463 & 1.91841 & 2.16665 & 2.49293 & 2.81171 & 3.04414 & 3.12958 \\
\hline & $\varphi_{0}(\mathrm{rad})$ & $\psi_{0}(\mathrm{rad})$ & $\alpha_{1}(\mathrm{rad})$ & $\alpha_{2}(\mathrm{rad})$ & $\alpha_{3}(\mathrm{rad})$ & $\alpha_{4}(\mathrm{rad})$ & $\Gamma$ \\
\hline Construction parameters & 1.24227 & $\begin{array}{r}-0.51977 \\
(5.76342)\end{array}$ & 0.38008 & 1.32488 & $\begin{array}{r}-1.49468 \\
(4.78851)\end{array}$ & 0.17467 & $\begin{array}{l}9.27502 \\
10^{-6}\end{array}$ \\
\hline
\end{tabular}




\subsection{Numerical example}

Picking the same interval $(2 \pi / 3<\varphi<4 \pi / 3)$, and the output function $\left(\psi=\varphi^{0.8}\right)$, seven design points in prescribed interval is given with respect to the Chebyshev spacing. The given design points and the calculated construction parameters are tabulated in Table 3 for each consecutive trials until the construction parameters are not changing in five decimals and Eq. (18) is satisfied. Also the plot of the designed mechanisms' objective function and its derivative in the design interval can be seen in Fig. 5 for trial 1 and trial 2 as the change between them is more visible.

When the results are compared with both of the previous methods, the values of the construction parameters $\left(\varphi_{0}, \psi_{0}, \alpha_{1}, \alpha_{2}, \alpha_{3}\right.$, $\alpha_{4}$ ) are too close again. However, by using Chebyshev approximation, at the end of the fourth trial the fitting errors are equalized for each of the extremum values. Note that, unlike the first method, design points in Chebyshev approximation are the points, where the fitting error reaches its extremum values in the design interval. Also the fitting error still vanishes at the six precision points.

\section{Discussion}

Although the synthesis methods shown in this study are applied only to spherical four bar mechanism, they are also valid for more common planar four bar function generators (similarly the joint axes are intersecting at infinity) as well as other mechanisms. On the other hand, it should be noted that the maximum number of independent parameters that can be synthesized in planar four bar function generators are limited to five $\left(\varphi_{0}, \psi_{0}, a, b, c\right)$ due to the ability of scaling (Fig. 6).

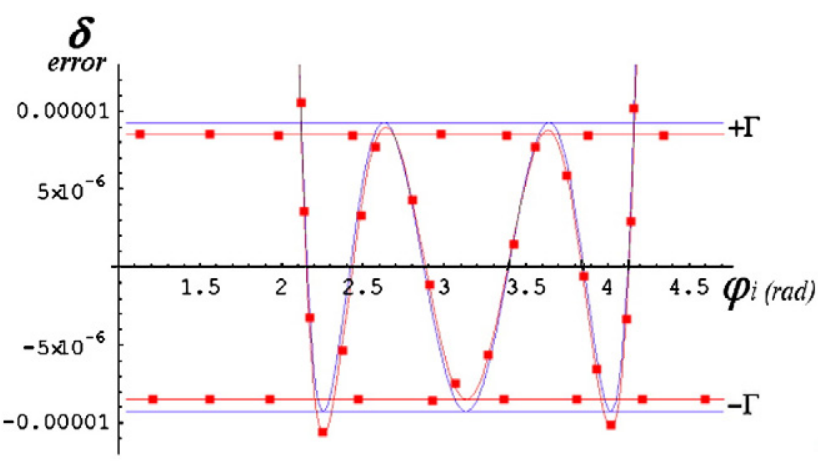

Trial 1

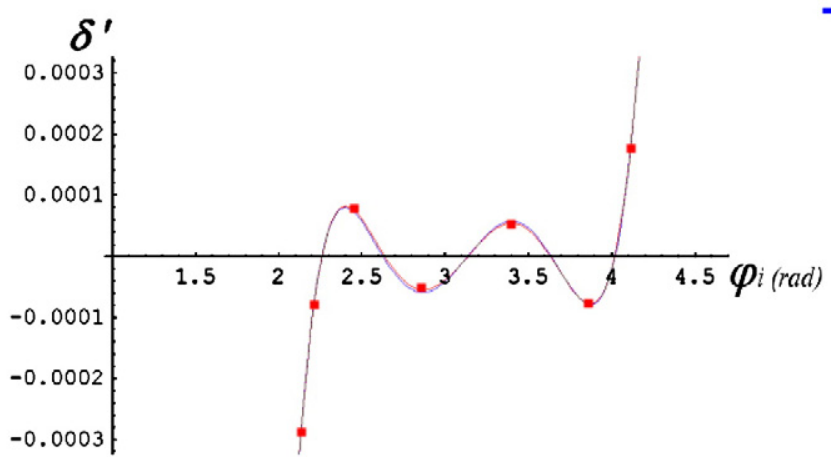

Trial 2

Fig. 5. Objective function of the designed spherical four bar mechanism and its derivative in Trial 1 and Trial 2 .

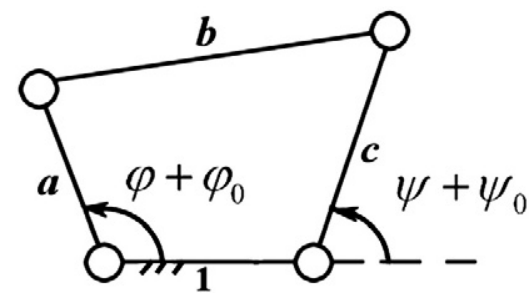

Fig. 6. Parameters of planar four bar mechanism function generator. 


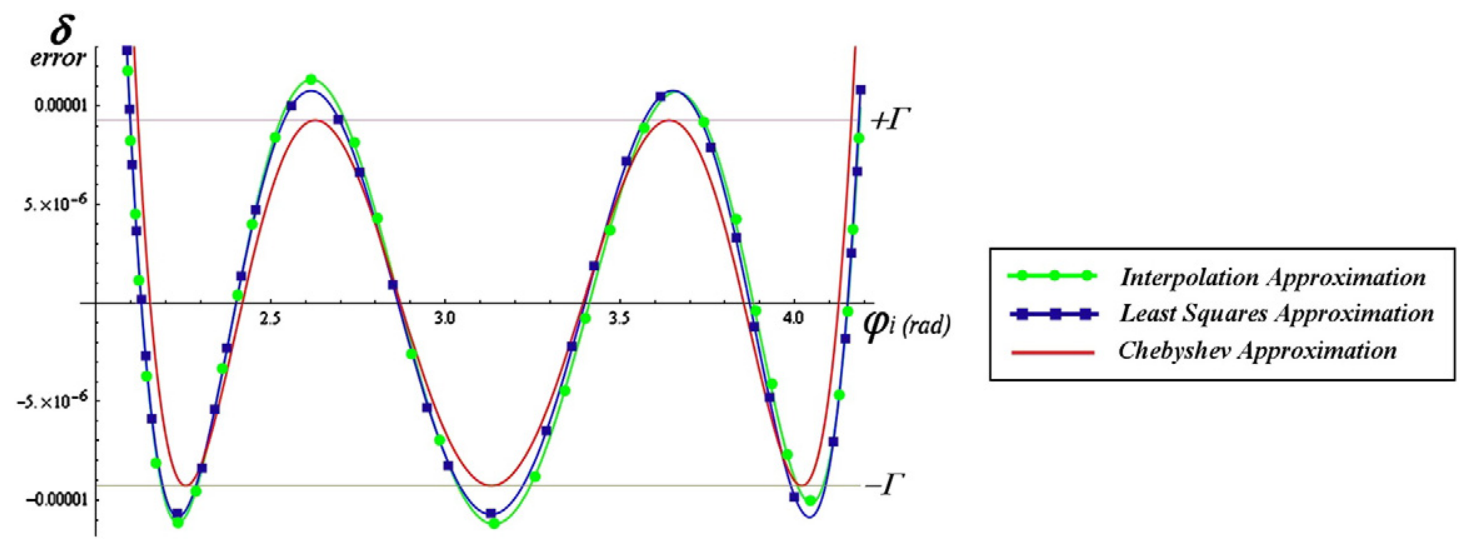

Fig. 7. Objective functions of the designed spherical four bar mechanisms for three different methods.

On the calculation side of this study, the reason behind the usage of the quaternion algebra in order to find the objective function of the mechanism is the simplicity of the tool for the rotations and the spherical four bar mechanism is just composed of rotational sequences. Moreover, the non-linear equations that are used to find the non-linear operators $\left\{\lambda_{k}\right\}_{1}^{6}$ in each example are solved numerically by using Mathematica software. Also, as an alternative method used by many authors in literature, the non-linear equation sets can be reduced into one equation with only one variable of non-linear operator in some order and again solved numerically in the end, if the order is high to solve analytically. After the results are acquired, the synthesized mechanisms are controlled in the simulation environment with respect to the calculated independent parameters. Note that as a different constraint additional conditions as rotability, branch and circuit defect elimination can also be controlled after the synthesis problem in other studies.

It is important that, the strategy followed in this work by using introduced methodologies is error based. The aim is not only to reduce the error but also to bind the error into some limits $\pm \Gamma$ so that the maximum absolute error in each interval between the precision points will be the same and oscillates back and forth in that interval. The Chebyshev approximation gave the best results in that manner with respect to the given examples. Finally, throughout the study the notion "design points" are used for the points that are given by the designer and the "precision points" are used for the points where the objective function of the synthesized mechanism takes zero values.

\section{Conclusion}

During this study, after the derivation of objective function by using quaternion algebra, function generation synthesis of spherical four bar mechanism for six independent parameters is introduced by using three kinds of methods as interpolation approximation, least squares approximation and Chebyshev approximation. Also, during the consecutive trials in Chebyshev approximation, a new approach is taken to renew the precision points $\varphi_{i}$. As it is not an easy task to find the precision points from the equations generated by the derivation of Eq. (21) with respect to $\varphi_{i}$, and equating them to zero, function of the derived equation is drawn in the design interval to compute $(n-2)$ roots. Afterwards, these roots are used as new $\varphi_{i}$ values with the previous boundary design points for the next trial. At the end of each method, a numerical example is given providing that the design intervals and the output function remains the same. Although values of the construction parameters $\varphi_{0}, \psi_{0}, \alpha_{1}, \alpha_{2}, \alpha_{3}$, and $\alpha_{4}$ of the three designed spherical four bar mechanisms are near, the generated objective functions and their fitting errors differ (Fig. 7).

As it can be seen in Fig. 7, interpolation approximation generates the highest fitting error, and also the error values at the extremums are not equal. On the other hand, least squares approximation decreased the highest fitting error value when compared with the interpolation approximation. However, the fitting error extremums are still not bounded in the same error values. Finally by using Chebyshev approximation, after the fourth trial, not only the maximum fitting error values are decreased with respect to the previous methods, but also the objective function starts to oscillate between an error bound of $\pm \Gamma$ with maximum absolute error $\Gamma$.

\section{References}

[1] R.S. Hartenberg, J. Denavit, Kinematic Synthesis of Linkages, McGraw-Hill, New York, 1964.

[2] J.R. Zimmerman, Four-precision synthesis of the spherical four-bar function generator, Mech. Mach. Theory 2 (1967) 133-139.

[3] R.I. Alizade, Synthesis of four-bar spherical mechanism on five parameters, J. Mech. Eng., Russian Academy of Science (ANR) 6 (1994) (in Russian).

[4] R.I. Alizade, O. Kilit, Analytic synthesis of function generating spherical four-bar mechanism for the five precision points, Mech. Mach. Theory 40 (7) (2005) $863-878$.

[5] K. Farhang, A. Midha, A.K. Bajaj, Synthesis of harmonic motion generation linkages part-I, function generation, ASME, J. Mech. Transm. Automat. Design 110 (1) (1988) 16-21.

[6] K. Farhang, Y.S. Zargar, Design of spherical 4R mechanisms: function generation for the entire motion cycle, ASME, J. Mech. Design 121 (1999) 521-528.

[7] A.V. Mohan Rao, G.N. Sandor, D. Kohli, A.H. Soni, Closed form synthesis of spatial function generating mechanism for the maximum number of precision points, J. Eng. Industry 95 (1973) 725-736. 
[8] A.P. Murray, J.M. McCarthy, A linkage map for spherical four position synthesis, ASME Tech. Conf. Boston. MA, 1995, pp. 833-844.

[9] R. Sancibrian, A. De-Juan, P. Garcia, A. Fernandez, F. Viadero, Optimal synthesis of function generating spherical and RSSR mechanisms, 12th IFToMM World Congress, Besançon (France), June 18-21, 2007, 2007.

[10] J. Jesus Cervantes-Sanchez a, Hugo I. Medellın-Castillo, Jose M. Rico-Martınez, Emilio J. Gonzalez-Galvan, Some improvements on the exact kinematic synthesis of spherical 4R function generators, Mech. Mach. Theory 44 (2009) 103-121.

[11] K. Kazerounian, R. Solecki, Mobility analysis of general bi-modal four-bar linkages based on their transmission angle, Mech. Mach. Theory 28 (3) (1993) 437-445.

[12] K.C. Gupta, A.S. Beloiu, Branch and circuit defect elimination in spherical four-bar linkages, Mech. Mach. Theory 33 (5) (1998) 491-504. 